\title{
The empathy deficit in health care
}

Dear Editor,

As an emergency medicine resident, an important part of my training is learning from mentors who practice in a way that I admire.

In addition to clinical acumen, if I had to choose one defining quality that my closest mentors share, it would be their high levels of empathy. Despite the challenges of our field and thousands of night shifts under their belts, these physicians continue to care deeply about every patient, no matter the complaint or demands on their time.

Empathy is the ability to put oneself in someone else's shoes, not just the shoes of those who represent a similar background, but, more importantly, the shoes of someone whose way of life is different from our own. Acting from an empathetic place means advocating for someone the way one would wish to be treated.

We tend to avoid discussing the empathy deficit in health care or perhaps do not recognize it. When we analyze an ethical scenario or a medical error, we can unpack some of the issues that produced the problem. We conclude, for example, with the "Swiss Cheese" model, that multiple misses caused the issue. We analyze training programs to determine if new doctors have the adequate knowledge to handle patient complaints. We look at physician burnout.

It is more rare to conclude that part of, or sometimes underlying a number of, the mishaps that occurred, there may have been an empathy issue-at an individual level among the health care providers involved-and at a systems level.

Just as going to the gym is required to get fit, exercising one's capacity for empathy requires empathetic action. It is a learned practice. Further, just as starting out at the gym is painful and exhausting, so is developing a meta-analysis of one's own behaviours and realizing that sometimes we are not as empathetic as we would like to be.

Performing a thorough and appropriate work-up for that intravenous drug user, elderly patient with dementia, or young person with serious mental health issues takes more effort and work than dismissing them.

I hope that those new to this field will rise to the challenge, myself included. From a patient perspective, it is their right as not only a patient but also, more fundamentally, a human being, to be treated with every ounce of empathy we can muster, no matter the circumstances.

Sincerely,

\section{Alicia Cundall, CCFP-EM Resident, Western University}

Keywords: empathy, empathy deficit, mentors 